\title{
Are Solar Active Regions with Major Flares More Fractal, Multifractal, or Turbulent than Others?
}

\author{
Manolis K. Georgoulis

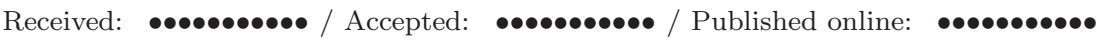

\begin{abstract}
Multiple recent investigations of solar magnetic field measurements have raised claims that the scale-free (fractal) or multiscale (multifractal) parameters inferred from the studied magnetograms may help assess the eruptive potential of solar active regions, or may even help predict major flaring activity stemming from these regions. We investigate these claims here, by testing three widely used scale-free and multiscale parameters, namely, the fractal dimension, the multifractal structure function and its inertial-range exponent, and the turbulent power spectrum and its power-law index, on a comprehensive data set of 370 timeseries of active-region magnetograms (17,733 magnetograms in total) observed by SOHO's Michelson Doppler Imager (MDI) over the entire Solar Cycle 23. We find that both flaring and non-flaring active regions exhibit significant fractality, multifractality, and non-Kolmogorov turbulence but none of the three tested parameters manages to distinguish active regions with major flares from flare-quiet ones. We also find that the multiscale parameters, but not the scale-free fractal dimension, depend sensitively on the spatial resolution and perhaps the observational characteristics of the studied magnetograms. Extending previous works, we attribute the flare-forecasting inability of fractal and multifractal parameters to $i$ ) a widespread multiscale complexity caused by a possible underlying self-organization in turbulent solar magnetic structures, flaring and non-flaring alike, and ii) a lack of correlation between the fractal properties of the photosphere and overlying layers, where solar eruptions occur. However useful for understanding solar magnetism, therefore, scale-free and multiscale measures may not be optimal tools for active-region characterization in terms of eruptive ability or, ultimately, for major solar-flare prediction.
\end{abstract}

Keywords: Active Regions, Magnetic Fields; Flares, Forecasting; Flares, Relation to Magnetic Field; Magnetic Fields, Photosphere; Turbulence

\footnotetext{
Research Center for Astronomy and Applied Mathematics (RCAAM), Academy of Athens, 4 Soranou Efesiou Street, Athens, Greece, GR-11527

email: manolis.georgoulis@academyofathens.gr
} 


\section{Introduction}

The ever-increasing remote-sensing capabilities of modern solar magnetographs have led to the undisputed conclusion that solar (active region in particular) magnetic fields exhibit an intrinsic complexity. "Complexity" is a term commonly used to describe an array of properties with one underlying characteristic: a scale-invariant, self-similar (fractal) or multiscale (multifractal) behavior. The measured photospheric magnetic fields in active regions are indeed multifractal (e.g. Lawrence, Ruzmaikin, and Cadavid, 1993, Abramenko, 2005), that is, consisting of a number of fractal subsets. As such, they are also fractal, with a fractal dimension equal to the maximum fractal dimension of the ensemble of fractal subsets.

Fractality is a mathematical property but with important physical implications. Scale-free or multiscale manifestations are thought to stem from an underlying self-organized, or self-organized critical (SOC), evolution in active regions. Self-organization refers to the internal, intrinsic reduction of the various parameters (also called degrees of freedom) of a nonlinear dynamical system, such as a solar active region, into a small number of important parameters that govern the system's evolution and, perhaps, its dynamical response (Nicolis and Prigogine, 1989). Assumptions on the nature of just these important parameters can lead to models of active-region emergence and evolution encapsulated in simplified cellular automata models (Wentzel and Seiden, 1992, Seiden and Wentzel, 1996, Vlahos et al., 2002, Fragos, Rantsiou, and Vlahos, 2004). Self-organized criticality, on the other hand, implies that the self-organized system evolves through a sequence of metastable states into a state of marginal stability with respect to a critical threshold. Local excess of the threshold gives rise to spontaneous, intermittent instabilities lacking a characteristic size (Bak, Tang, and Wiesenfeld 1987, Bak, 1996).

The intrinsic self-organization in solar active regions may be attributed to the turbulence dominating the emergence and evolution of solar magnetic fields. Tangled, fibril magnetic fields rising from the convection zone can be explained via Kolmogorov's theory of fluid turbulence (e.g., Brandenburg et al., 1990. Longcope, Fisher, and Pevtsov, 1998, Cattaneo, Emonet, and Weiss, 2003, and others). Turbulence in the generation and ascension of solar magnetic fields leads to turbulent photospheric flows (e.g., Hurlburt, Brummel, and Toomre, 1995). Thus, the turbulent photosphere is viewed as a driver that gradually but constantly perturbs an emerged magnetic-flux system, such as an active region, dictating self-organization in it and possibly forcing it toward a marginally stable, SOC state (e.g., Vlahos and Georgoulis, 2004). Turbulent action does not cease in the photosphere, but it extends into the solar corona. However, coronal low- $\beta$ turbulence may not be the Kolmogorov fluid turbulence applying to the high- $\beta$ plasma of the convection zone and the photosphere. Instead, it might be an intermittent magnetohydrodynamic (MHD) turbulence (Kraichnan, 1965. Biskamp and Welter, 1989).

Fractal, multifractal, and turbulent properties of photospheric active-region magnetic fields have been intensely studied in recent years. Fractality is traditionally investigated via the fractal dimension, often inferred using box-counting 
techniques (e.g., Mandelbrot, 1983). Box-counting is also used for multifractal studies in space and time (e.g., Evertsz and Mandelbrot, 1992), involving also generalized correlation dimensions (Georgoulis, Kluiving, and Vlahos, 1995. Kluiving and Pasmanter, 1996). A commonly used multifractal method that does not require box counting is the calculation of the multifractal structure function spectrum (Frisch, 1995). Moreover, a practical method for quantifying turbulence is the calculation of the turbulent power spectrum, stemming from the original work of Kolmogorov (1941). If the power spectrum shows a power law over a range of scales, perceived as the turbulent inertial range, its slope determines whether the inferred turbulence is Kolmogorov-like (scaling index $\approx-5 / 3$ ) or Kraichnan-like (scaling index $\approx-3 / 2$ ) if either of these two applies.

Multiple studies on fractality, multifractality, and turbulence in photospheric active-region magnetic fields have raised claims that flaring active regions exhibit distinct, distinguishable complexity. These works might lead to the impression that fractal, multifractal, or turbulent measures hold significant flare-predictive capability or, at least, they might be used to identify flaring active regions before they actually flare. To summarize some of these works, Abramenko et al. (2003) suggested that a "peak in the correlation length might be a trace of an avalanche of coronal reconnection events". McAteer, Gallagher, and Ireland (2005) reported that "solar flare productivity exhibits an increase in both the frequency and GOES X-ray magnitude of flares from [active] regions with higher fractal dimension". Further, Abramenko (2005) found that "the magnitude of the power index at the stage of emergence of an active region ... reflects its future flare productivity when the magnetic configuration becomes well evolved", while Georgoulis (2005) reported that "the temporal evolution of the [inertial-range] scaling exponents in flaring active regions probably shows a distinct behavior a few hours prior to a flare". More recently, Conlon et al. (2008) worked on a sample of four active regions and reported evidence for a "direct relationship between the multifractal properties of the flaring regions and their flaring rate", while Hewett et al. (2008), reporting on "preliminary evidence of an inverse cascade in active region NOAA 10488" found a "potential relationship between energy [power-spectrum] scaling and flare productivity". Many of these works are also reviewed by McAteer, Gallagher, and Conlon (2010).

If the above findings are confirmed, they may well lead to notable improvements in our physical understanding of active regions and in highlighting possible differences between flaring (that is, hosting major flares) and non-flaring (that is, hosting only sub-flares) regions. In Georgoulis (2005) we studied three different scale-free and multiscale parameters, namely, the fractal dimension, the spectrum of generalized correlation dimensions, and the structure-function spectrum and its inertial-range exponents over a limited magnetogram sample of six active regions, three of them hosting at least one major flare (M- or X-class in the GOES X-ray 1-8 $\AA$ flare classification scheme). In one case of a Xflaring active region - NOAA active region (AR) 10030 with an X3 flare at the time of the observations - we noticed a sharp preflare increase of the inertialrange exponent of the structure functions followed by a significant $(\approx 20 \%$ and much above uncertainties), permanent decrease after the flare. We suggested 
that this analysis should be repeated on a much larger sample of both flaring and non-flaring regions to determine whether this behavior was incidental.

In this study we analyze a comprehensive sample of 370 timeseries of activeregion magnetograms, with each timeseries corresponding to a different active region. In this sample, 77 active regions hosted at least one $\mathrm{M}$ - or X-class flare during the observations and they are considered flaring (17 X-class flaring, 60 M-class flaring), while the remaining 293 active regions were not linked to major flares and are hence considered non-flaring. We calculate three of the most promising scale-free and multiscale measures on this data set, namely, the fractal dimension, the multifractal structure function spectrum, and the turbulent power spectrum. A detailed description of the data and techniques follows in Section 2 In Section 3 we test the sensitivity of the calculated parameter values on the spatial resolution of the studied magnetogram. A statistical analysis of the active-region sample is performed in Section 4 while Section 5 summarizes the study, discusses the results, and outlines our conclusions.

\section{Design of the Study}

\subsection{Magnetogram Data}

Our active-region sample has been constructed using data from the MichelsonDoppler Imager (MDI: Scherrer et al., 1995), onboard the Solar and Heliospheric Observatory (SOHO) mission. We acquired the entire MDI magnetogram archive from mid-1996 to late-2005. The archive consists of full-disk line-of-sight solar magnetograms taken at a 96 -minute cadence with a linear pixel size of $\approx 1.98$ $\operatorname{arcsec}($ a mean $\approx 1440 \mathrm{~km}$ at solar disk center, depending on Sun-Earth distance). This analysis uses purely line-of-sight, Level 1.5 SOHO/MDI magnetic field measurements that are known (Berger and Lites, 2003) to underestimate sunspot and plage fields (more recent, Level 1.8.2 sensitivity corrections to the MDI full-disk magnetograms are not used in this study because our full-disk magnetogram dataset was constructed in 2007 and the recalibrated magnetograms were posted in December 2008). Nonetheless, we have avoided applying any additional corrections to the data to avoid a possible impact on the morphological characteristics of the regions studied, since fractal and multifractal analysis highlights exactly these characteristics. To reduce the impact of projection effects acting on the magnetic field vector, we restrict our study to a $60^{\circ}$ longitudinal region centered on the central solar meridian. Use of this zone introduces a systematic underestimation in the normal magnetic field component by a factor up to $\approx(1-\cos (\theta)) \simeq 0.14$, or $14 \%$, at the Equator, for a central meridian distance $\theta=30^{\circ}$ approximated by the angular difference between the local normal and the line of sight for an observer at Earth. Conventionally, this underestimation factor is deemed tolerable when the line-of-sight field component is used as a proxy of the normal field component. Within this $60^{\circ}$ meridional zone we identified and extracted active regions using our automatic active-region identification algorithm (ARIA), detailed in LaBonte, Georgoulis, and Rust (2007) and in Georgoulis, Raouafi, and Henney (2008). Our ARIA extracts portions of 




Figure 1. Pictorial output of our ARIA, applied to a full-disk SOHO/MDI magnetogram acquired on 29 October 2003. A $60^{\circ}$ meridional zone centered on the central meridian is indicated by the thick dashed brackets. Three active regions fulfilled our selection criteria, namely, NOAA ARs 10486, 10487, and 10488. The portion of the disk found to include each region is shown by the thin dashed circles - the actual portion extracted for each region is shown by the circumscribed squares. The NOAA labels for each region are provided automatically. The flux-weighted centroids for each active region are represented by white crosses.

the solar disk corresponding to active regions by means of pattern recognition in which the unit length is one supergranular diameter (40 arcsec). A typical example is shown in Figure 1. An active region is chosen for further study if its flux-weighted centroid, shown by the white crosses in the selected regions of Figure 1, falls within the above-mentioned $60^{\circ}$ meridional zone. Notice, for example, that NOAA AR 10487 is selected in Figure 1 because its flux-weighted centroid lies within the above zone; parts of it, however, extend beyond this area.

Besides the automatic active-region selection process, each selected magnetogram (out of a total of 17,733 ) was manually examined to exclude portions of other active regions that might intrude in the field of view. For example, NOAA AR 10486 in Figure 1 is included in its selection circle, but the square circumscribed on this circle crops sizable parts of NOAA ARs 10489 and 10491 in its northwestern edge. These parts have been excluded in the subsequent analysis. Generally our ARIA performs quite well in distinguishing active regions but few incidences such as the above have been noted, especially in cases of densely populated active-region complexes, or "nests", such as the one be- 


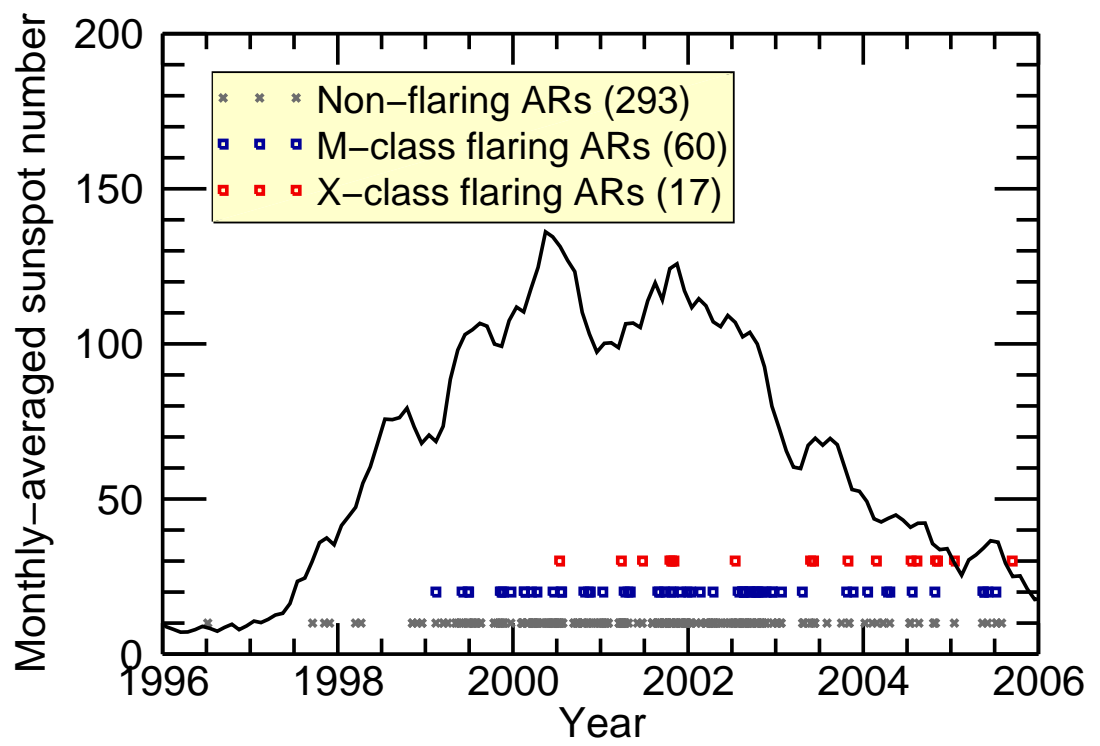

Figure 2. Temporal distribution of our sample of 370 active regions over Solar Cycle 23. Shown are the median observation times (color symbols) of each region together with the monthly-averaged sunspot number (curve).

lieved to have occurred during the October-November "Halloween" 2003 period (Zhou et al., 2007). For our analysis, ARIA uses a maximum tolerated magneticflux imbalance of $50 \%$ in a given active region and a minimum active-region linear size of one supergranular diameter. For each of the 370 selected active regions we created a timeseries consisting of up to $\approx 60$ magnetograms taken every 96 minutes corresponding to the approximately four-day period needed for each active region to traverse the $60^{\circ}$-analysis zone.

To document the major flare history for each active region we browsed $i$ ) NOAA's GOES X-ray archive and ii) the Yohkoh/HXT flare catalog (available online, at http://gedas22.stelab.nagoya-u.ac.jp/HXT/catalogue/). From the total of 370 active regions, 77 were unambiguously found to have hosted at least one M-class or X-class flare while within $\pm 30^{\circ}$ of the central meridian, with a total of $24 \mathrm{X}$-class flares and $87 \mathrm{M}$-class flares. Our active-region sample roughly covers Solar Cycle 23, as shown in Figure 2 The solar cycle is represented by a 5-point running mean of the monthly-averaged sunspot number obtained by the Solar Influences Data Analysis Center (SIDC) of the Royal Observatory of Belgium.

In addition to the above SOHO/MDI sample, our analysis includes three nearly simultaneous magnetograms of NOAA AR 10930, observed on 11 December 2006. The line-of-sight components of these magnetograms are depicted in Figure 3. Figure 33 shows the Level 1D magnetogram (preferred over Level 2 data in order to better qualify for comparison with SOHO/MDI Level 1.5 data) acquired by the Spectropolarimeter (SP: Lites, Elmore, and Streander, 2001) of the Solar Optical Telescope (SOT) onboard the Hinode satellite and has a very 

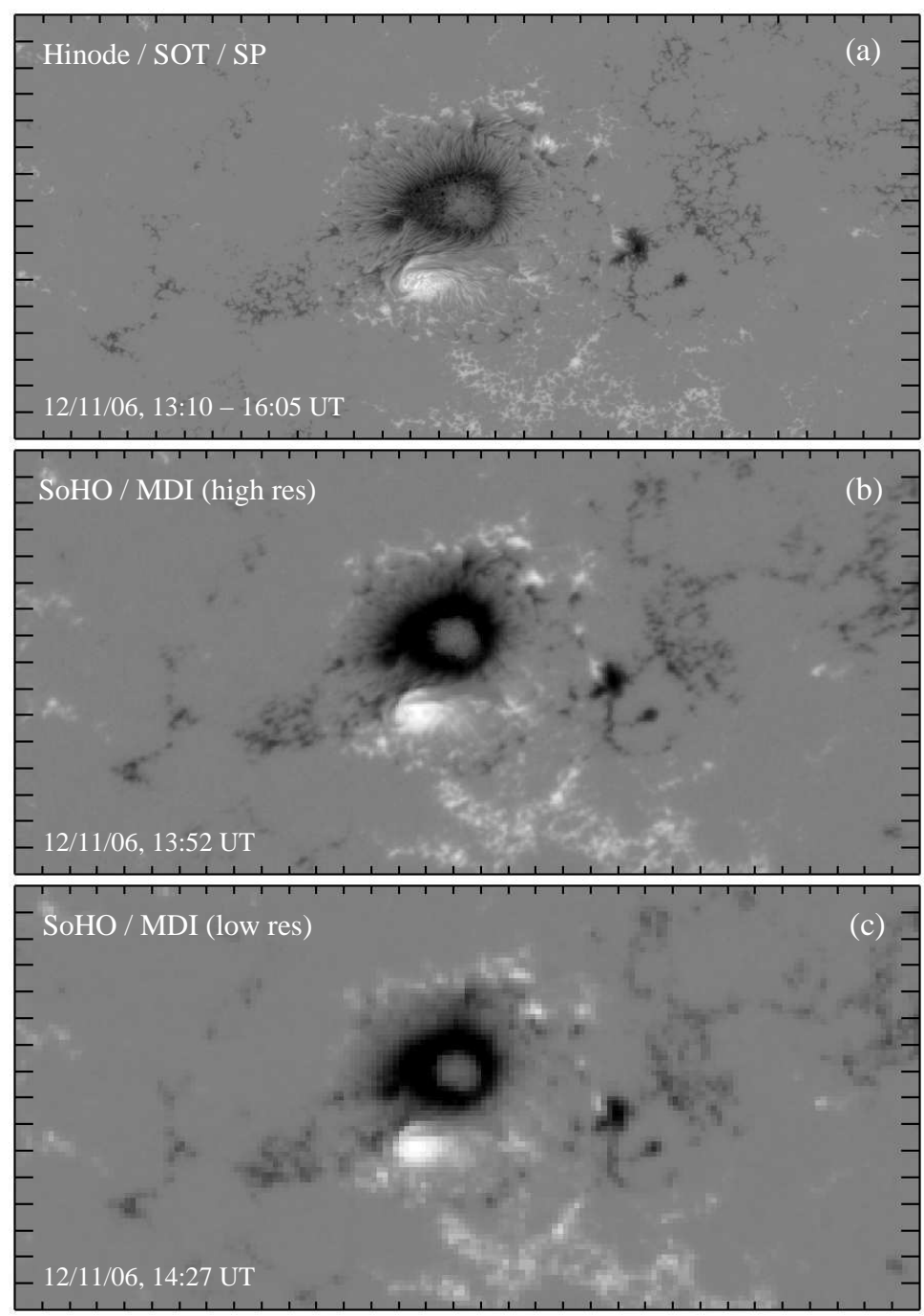

Figure 3. Nearly simultaneous, coaligned magnetograms of NOAA AR 10930, acquired on 11 December 2006: observations are from (a) Hinode's SOT/SP, (b) SOHO/MDI high-resolution, partial disk magnetograph, and (c) SOHO/MDI full-disk magnetograph. The linear pixel sizes are $0.158 \operatorname{arcsec}, 0.605 \operatorname{arcsec}$, and $1.98 \operatorname{arcsec}$ for (a), (b), and (c), respectively. Shown is the line-of-sight magnetic field component saturated at $\pm 2.5 k G$ (a), $\pm 1 \mathrm{kG}$ (b), and $\pm 1.8 \mathrm{kG}$ (c). Tic mark separation in all images is 10 arcsec.

high spatial resolution $(\approx 0.32 \operatorname{arcsec})$ with a linear pixel size of $\approx 0.158$ arcsec. Figure $3 \mathrm{~b}$ shows the respective magnetogram taken by the SOHO/MDI highresolution, partial-disk magnetograph, with a coarser linear pixel size of 0.605 arcsec. Figure 3r shows the Level $1.5 \mathrm{SOHO} / \mathrm{MDI}$ magnetogram extracted by a full-disk measurement with a much coarser linear pixel size of 1.98 arcsec. The three magnetograms have been initially coaligned by means of the pointing information provided separately for each. To further correct and deal with small 
pointing inconsistencies, coalignment has been completed by a rigid displacement (translation) over the $\mathrm{E}-\mathrm{W}$ and the $\mathrm{N}-\mathrm{S}$ axes. Displacements are determined by the peak of the cross-correlation function between a given pair of images, with cross-correlation functions inferred by means of fast Fourier transforms.

The three distinctly different spatial resolutions of the magnetograms of Figure 3 will be useful when testing the sensitivity of our scale-free and multiscale parameters to varying spatial resolution (Section 3).

\subsection{Scale-free and Multiscale Techniques}

The first parameter that we calculate is the scale-free, two-dimensional fractal dimension $\left[D_{0}\right]$ of the active-region magnetograms. To calculate $D_{0}$ we cover the magnetogram field-of-view with a rectangular grid consisting of square boxes with linear size $[\lambda]$ and area $\lambda \times \lambda$. Assuming that the field of view is a square with linear size $L$ and area $L \times L$, the number of boxes needed to cover it is $(L / \lambda)^{2}$. Each of the boxes will have a dimensionless area $\varepsilon^{2}$, where $\varepsilon=\lambda / L$. Of the total $(L / \lambda)^{2}$ boxes we count those that include part of the boundary of a strong-field magnetic configuration (see below for the adopted strong-field definition). Then, varying the box size $\lambda$ or, equivalently, the dimensionless size $\varepsilon$, we obtain different numbers $[N(\varepsilon)]$ of information-carrying boxes. Correlating the various numbers $N(\varepsilon)$ with the respective box sizes $\varepsilon$, we obtain the scaling relation

$$
N(\varepsilon) \propto(1 / \varepsilon)^{D_{0}} .
$$

For a non-fractal, Euclidean structure embedded on a plane we have $N(\varepsilon)=$ $(L / \lambda)^{2}=(1 / \varepsilon)^{2}$, so $D_{0}=2$. If $D_{0}<2$, we have fractal structures with a scalefree, incomplete filling of the field of view. The stronger the departure of $D_{0}$ from its Euclidean value of 2, the finer the structure exhibited by the studied magnetic configuration. For $D_{0} \leq 1$ in a two-dimensional fractal, the structures are typically scattered into a scale-free hierarchy of small "islands', resembling what is known as fractal dust (e.g. Schroeder, 1991).

We infer the fractal dimension $D_{0}$ by a least-squares best fit of the scaling relation of Equation (11). The uncertainty associated with the value of $D_{0}$ is equal to the uncertainty of the regression fit. To guarantee a reliable inference of $D_{0}$, we demand that the dynamical range represented by the least-squares fit exceeds one order of magnitude. A very small fraction of magnetograms of nonflaring active regions $(\simeq 0.6 \%$, or 111 magnetograms) happen not to comply with this requirement because of the regions' simplicity and scattered configurations; these magnetograms have been excluded from the analysis. Nonetheless, each of the 370 active regions of our sample fulfills the requirement with at least one magnetogram.

McAteer, Gallagher, and Ireland (2005), relying on a substantial data set of $\approx 10^{4}$ active regions, first reported that flaring regions have fractal dimensions $D_{0} \geq 1.2$. Their finding was statistical, of course, meaning that the $D_{0} \geq 1.2$ condition should be viewed as a necessary, but not sufficient, condition for major flare productivity. They also concluded, and we test their result here, that intensely flaring active regions showed statistically higher fractal dimensions. 
To better compare with the results of McAteer, Gallagher, and Ireland (2005), we follow their criterion when outlining the boundaries of active regions: first, we use a threshold of $50 \mathrm{G}$ in the strength of the line-of-sight field component in order to define the outer contours of strong-field magnetic patches. Then we impose a lower limit of 20 pixels for the length of each contour, thus rejecting very small patches that could as easily belong to the quiet Sun. As an additional condition, we impose a lower flux limit of $10^{20} \mathrm{Mx}$ for each patch. For the MDI low-resolution data this is nearly equivalent to saying that at least 100 pixels within the patch should have a line-of-sight field strength of at least $50 \mathrm{G}$, which is our threshold. Of course, a selected patch can contain fewer than 100 (but more than 20) pixels but with larger field strength in order to satisfy the flux condition.

The second parameter that we calculate is the multifractal structure function spectrum (Frisch, 1995). The spectrum is given by

$$
S_{q}(r)=\left\langle|\Phi(\mathbf{x}+\mathbf{r})-\Phi(\mathbf{x})|^{q}\right\rangle
$$

and it does not rely on box-counting or thresholding, contrary to $D_{0}$. Instead, on the magnetic-flux distribution $[\Phi(\mathbf{x})]$ of the active-region photosphere we define a displacement vector $[\mathbf{r}]$, also called the separation vector, and calculate the variation of the flux at this displacement. The variation is then raised to the power $q$, where $q$ is a real, preferably positive number called the selector. Spatial averaging $(\langle\rangle)$ of the structure function over $\mathbf{x}$ and all possible orientations of $\mathbf{r}$ gives rise to a unique, positive value $S_{q}(r)$ of the structure function for a given pair $(r=|\mathbf{r}|, q)$. The resulting spectrum involves a range of $r$-values and a fixed value of $q$; different spectra are obtained for different $q$-values.

The multifractal structure function is designed to highlight the intermittency present in a magnetic-flux distribution (Abramenko et al., 2002, 2003). In the case of a multifractal, intermittent flux distribution, the structure function $\left[S_{q}(r)\right]$ exhibits a power law

$$
S_{q}(r) \propto r^{\zeta(q)}
$$

within a range of displacements, often referred to as the turbulent inertial range. The upper and lower extremes of the $r$-range correspond to, respectively, the maximum size of structures entering the inertial range and the scale over which ideal cascading of energy to smaller scales breaks down by dissipative effects. Higher values of the inertial-range scaling index $\zeta(q)$ indicate a higher degree of intermittency, with $\zeta(q)=q / 3$ implying absence of intermittency.

Abramenko, Yurchyshyn, and Wang (2008) studied the structure function spectrum and implemented an additional suggestion by Frisch (1995) to examine the flatness function $f=S_{6}(r) / S_{3}(r)^{3} \propto r^{-\delta}$, where $\delta$ is viewed as the intermittency index with higher values implying a higher degree of intermittency. They concluded that photospheric and coronal magnetic fields are both intermittent, with the intermittency in the photosphere preceding that in the corona and the corona responding to photospheric increases of intermittency. In their analysis, they used data from the same active region we show in Figure 3 . 
namely, NOAA AR 10930, observed by Hinode/SOT/SP and SOHO/MDI highresolution magnetographs, although not on the same day with the data used in this study.

Georgoulis (2005) used the structure function $S_{q}(r)$ of Equation (3) and the inertial-range scaling exponent $\zeta(q)$ to show that the photospheric magnetic fields of the six active regions of the study were indeed multifractal and intermittent, departing strongly from $\zeta(q)=q / 3$. More importantly, Georgoulis (2005) presented an example of a X3 flare that occurred in NOAA AR 10030, where the intermittency peaked $\approx 1-2$ hours prior to the flare and decreased sharply after the flare. The decrease was permanent and $\zeta(q)$ continued decreasing $\approx 1.5$ hours later, when an M1.8 flare also occurred in the active region (Figure 7 of Georgoulis, 2005). The change in the degree of intermittency was best seen for a selector $q \in(3,3.5)$. Here we investigate whether this distinct behavior, seen in only one example, is part of a systematic tendency. To this purpose we study the temporal evolution of the scaling index $\zeta(q=3)$, inferred by a least-squares best fit of the scaling relation of Equation (3). The uncertainty of the regression fit is treated as the uncertainty of the value of $\zeta(3)$. For the uncertainty of the $\zeta(3)$-differences we simply propagate the uncertainties of the two $\zeta(3)$-values that make these differences.

The third parameter we study is the turbulent power spectrum $[E(k)]$. In the case of a turbulent flux system, there exists an inertial range of wavenumbers $k$ reflected on a power-law form for $E(k)$ :

$$
E(k) \propto k^{-\alpha},
$$

where $\alpha$ is the inertial-range exponent. We demand at least one order of magnitude as the dynamical range of the least-squares best fit used to infer $\alpha$ and we attribute the uncertainty of the fit to the uncertainty of $\alpha$. Like $S_{q}(r)$ and unlike $D_{0}$, no box-counting or thresholding is required to infer $\alpha$.

Abramenko (2005) suggested that the $k$-range corresponding to length scales $r \in(3,10) \mathrm{Mm}$ should be scaled according to the representative $\alpha$-value for high-resolution MDI magnetograms. Although we use MDI low-resolution data for this analysis, we follow Abramenko's suggestion and make sure that the $(3,10) \mathrm{Mm}$ scale range is included in the power-law fit. We also attempt to test the conclusion of Abramenko (2005), relying on a sample of 16 high-resolution MDI active-region magnetograms, that the inertial-range exponent $\alpha$ reflects the future flare productivity of an active region, with larger $\alpha$-values implying a higher flare index (Figure 8 of Abramenko, 2005). It is also important to mention here Abramenko s (2005) conclusion that the scaling index $\alpha$ is not particularly useful for the prediction of imminent flares in the studied active regions.

A typical calculation of the fractal dimension $D_{0}$, the inertial-range scaling index $\zeta(3)$, and the scaling exponent $\alpha$ of the turbulent power spectrum for the low-resolution MDI magnetogram of Figure 3 r is depicted in Figure 4

With the exception of the turbulent power spectrum, Georgoulis (2005) used all of the above methods, including the spectrum of multifractal generalized correlation dimensions. The latter showed that the six active regions of the sample had clearly multifractal photospheric magnetic fields. The method is not 

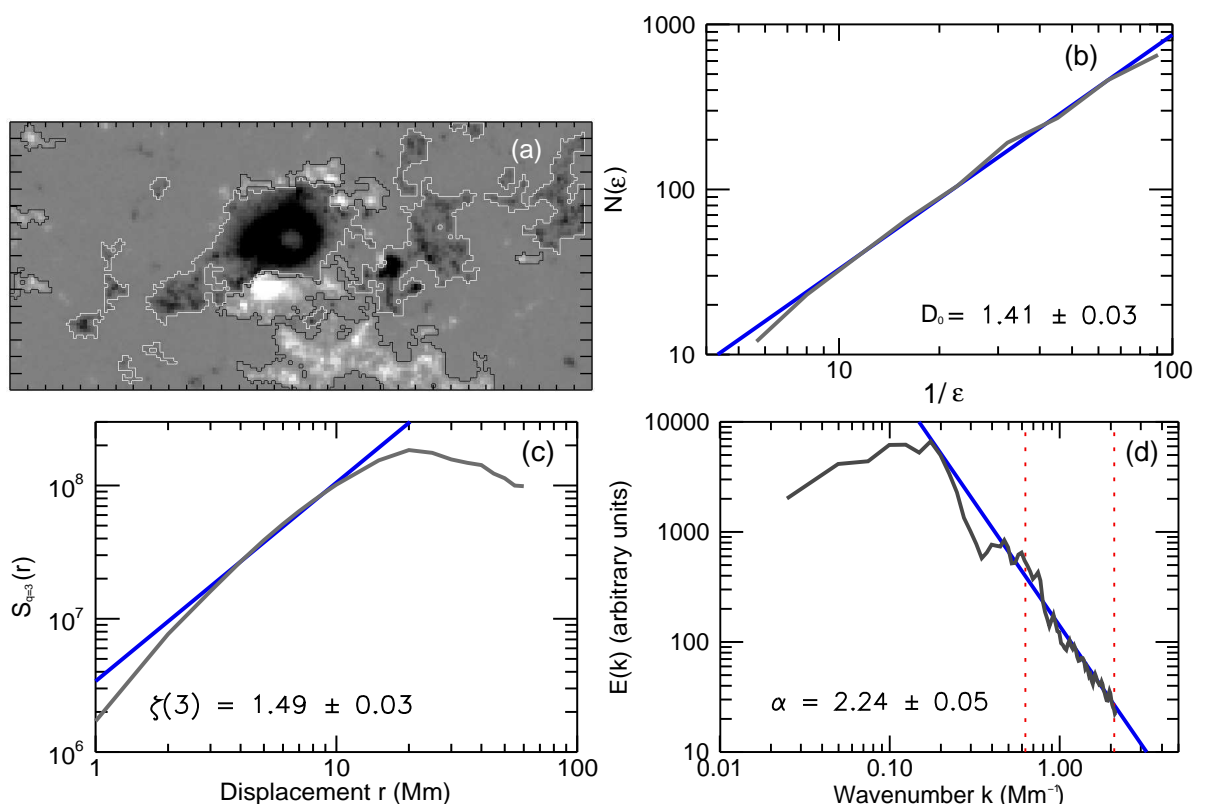

Figure 4. Typical calculation of the scale-free and multiscale parameters that will be used to quantify the complexity of solar active regions. The example refers to the low-resolution $\mathrm{SOHO} / \mathrm{MDI}$ magnetogram of Figure 3 , shown in (a). The magnetogram is saturated at \pm 1 $\mathrm{kG}$. Contours indicate the areas where the line-of-sight field strength exceeds the threshold of 50 G. Tic mark separation is 10 arcsec. Also shown are (b) the scaling relation of Equation (1D) yielding the fractal dimension $D_{0}$, (c) the structure function $S_{q=3}(r)$ of Equation (2), yielding the inertial-range scaling index $\zeta(3)$, and (d) the turbulent power spectrum of Equation (4), yielding the inertial-range scaling exponent $\alpha$. The two dotted lines indicate the wavenumbers of the desired length scales of $10 \mathrm{Mm}(k<1)$ and $3 \mathrm{Mm}(k>1)$. Gray curves in b, c, and d correspond to $N(\varepsilon), S_{q=3}(r)$, and $E(k)$, respectively, while the blue lines are the respective least-squares best fits.

used in this study, however, because in Georgoulis (2005) it clearly failed to distinguish between flaring and non-flaring regions even for the handful of active regions studied.

The objective of this work is to test the flare-predictive capability of the above - reported to be promising - parameters. This capability will be tested by comparison with the capability of a standard, traditional parameter reflecting the size of active regions, namely, the unsigned magnetic flux

$$
\Phi_{t o t}=\int_{S}\left|B_{n}\right| \mathrm{d} S
$$

where $B_{n}$ is the normal magnetic field component (approximated by the lineof-sight field component near disk center) over the magnetograms' field of view $[S]$. Flaring regions are statistically more flux-massive than non-flaring ones. As a result, the unsigned flux is considered a "standard" flare forecasting criterion that, however, is not without limitations (e.g., Leka and Barnes, 2003), especially when it comes to flux-massive, but quiescent, active regions. For a 
Table 1. Values and uncertainties (in parentheses) of the unsigned magnetic flux $\Phi_{\text {tot }}$, th fractal dimension $D_{0}$, the inertial-range scaling exponent $\zeta(3)$, and the power-spectrum index $\alpha$ for the three nearly simultaneous and coaligned magnetograms of NOAA AR 10930 acquired on 11 December 2006 (Figure 3).

\begin{tabular}{ccccccc}
\hline $\begin{array}{c}\text { Ref. } \\
\text { (Fig.) }\end{array}$ & $\begin{array}{c}\text { Observation } \\
\text { Time (UT) }\end{array}$ & $\begin{array}{c}\text { Pixel size } \\
(\operatorname{arcsec})\end{array}$ & $\begin{array}{c}\Phi_{\text {tot }} \\
\left(\times 10^{22} \mathrm{Mx}\right)\end{array}$ & $D_{0}$ & $\zeta(3)$ & $\alpha$ \\
\hline 3. & $13: 10-16: 05$ & 0.158 & 2.85 & $1.54(0.04)$ & $1.35(0.03)$ & $3.00(0.02)$ \\
3. & $13: 52: 01$ & 0.605 & 2.12 & $1.43(0.02)$ & $1.67(0.04)$ & $3.32(0.04)$ \\
3. & $14: 27: 01$ & 1.980 & 3.60 & $1.41(0.03)$ & $1.49(0.03)$ & $2.24(0.05)$ \\
\hline
\end{tabular}

parameter to be characterized as having a significant predictive capability, it has to perform better than the unsigned flux.

\section{Dependence of Scale-Free and Multi-Scale Parameters on the Spatial Resolution}

Prior to analyzing our extensive MDI dataset we perform a sensitivity test of the parameters introduced in Section 2.2 to the spatial resolution of the studied magnetogram. Ideally, a parameter reliable enough to distinguish flaring from non-flaring active regions should be fairly insensitive to varying spatial resolution. If this were not the case, then one should at least be able to model the parameter's variations with changing resolution. If these conditions are not fulfilled, likely threshold values of the parameter that one may use to identify flaring regions before they flare are resolution-dependent and, as a result, instrument-dependent.

We use the three magnetograms of NOAA AR 10930 shown in Figure 3 to test the parameter values on data with varying spatial resolution. The results are provided in Table 1 for the parameters $\Phi_{\text {tot }}$ [Equation (5)], $D_{0}$ [Equation (10)], $\zeta(q=3)$ [Equation (3)], and $\alpha$ [Equation (4)]. Our findings can be summarized as follows:

i) Despite coalignment and near simultaneity, the unsigned magnetic flux $\Phi_{\text {tot }}$ shows distinct differences for the three different magnetograms: the MDI low-resolution magnetogram (Figure 3 ; ) shows $\approx 25 \%$ larger flux than the SOT/SP magnetogram (Figure 3a), while the MDI high-resolution magnetogram (Figure $3 \mathrm{~b}$ ) shows $\approx 28 \%$ less flux than the SOT/SP magnetogram. Some additional testing, not shown here, has been performed in an attempt to explain these large differences. In particular, attempting to degrade the highest-resolution magnetograms (SOT/SP and high-resolution MDI) to simulate situations of a larger pixel size ( 2 arcsec and more) we find a very weak decreasing trend for the unsigned flux that reaches up to $\approx 3.5 \%$ between the highest- (original) and the lowest- (degraded) resolution magnetogram. Likely, therefore, the $\approx 30 \%$ flux difference is not due to the different spatial resolution. Similar results are obtained when the spatial resolution of 
the SOT/SP and high-resolution MDI data is decreased by resampling. We cannot be certain about the source(s) of the discrepancy but, given that we have used SP and MDI data of similar calibration levels, one might attribute this discrepancy to differences in the observations, processing, and calibration between the SP and the two MDI magnetographs. Discussing those differences exceeds the scope of this work.

ii) The values of the scale-free fractal dimension $D_{0}$ are fairly consistent, despite the widely different spatial resolution and the different instruments. One notices a significant (i.e., beyond error bars) decreasing tendency for decreasing resolution but the overall decrease for the $\approx 13$-fold difference in resolution between the SOT/SP and the low-resolution MDI data is only $\approx 8.5 \%$. This is probably because the fractal dimension qualitatively highlights the morphological complexity of the studied self-similar structure that is being reflected adequately on seeing-free (SOT/SP and MDI) magnetograms largely regardless of spatial resolution and magnetic flux content.

iii) The values of the multiscale inertial-range scaling exponent $\zeta(3)$ and the power-spectrum scaling index $\alpha$ appear strongly dependent on the spatial resolution and/or other instrumental characteristics. The variation of both indices is not even monotonic, with their peak values corresponding to the intermediate case of the high-resolution MDI magnetogram. One might speculate that this happens because different spatial resolution changes quantitatively, but perhaps not qualitatively, the multiscale character of the data. In this sense, despite different parameter values, all three magnetograms of NOAA AR 10930 show significant intermittency $(\zeta(3) \gg 1$, with lack of intermittency reflected on $\zeta(3)=1$ ) and non-Kraichnan/non-Kolmogorov turbulence $(\alpha \gg 3 / 2$ and $5 / 3$, respectively).

The susceptibility of the multiscale parameters $\zeta(3)$ and $\alpha$, but not of the scale-free parameter $D_{0}$, to the spatial resolution implies caution when utilizing multiscale parameters to distinguish flaring from non-flaring active regions. At the very least, quantitative results in this case should not be generalized to different data sets. We therefore stress that the results described in the next Sections for the multiscale parameters correspond exclusively to the MDI fulldisk spatial resolution of $\approx 2$ arcsec.

\section{Comparison of Parameters for Flaring and Non-Flaring Active Regions}

To determine whether any of the fractal or multifractal parameters $D_{0}, \zeta(3)$, and $\alpha$, including $\Phi_{\text {tot }}$ as a reference, can distinguish flaring from non-flaring active regions, we perform two tests: a more stringent one, that compares the preflare (96 minutes in advance, at most, per the cadence of the full-disk MDI magnetograms) values of the parameters for flaring active regions to the peak values of the parameters for non-flaring regions, and a more liberal one, that compares the peak values of the parameters for both flaring and non-flaring regions. Finding a distinguishing pattern in the first test would mean that the studied parameter may have a short-term predictive capability. If the first test fails but the second 

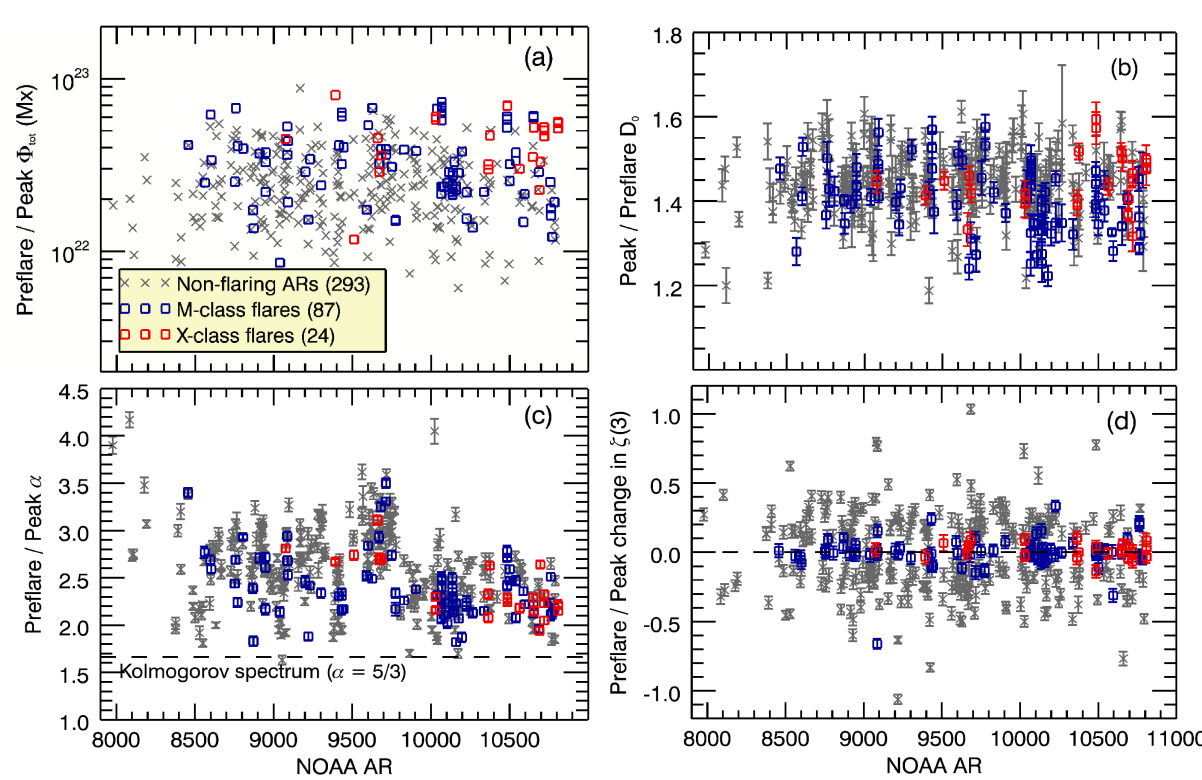

Figure 5. Comparison between the preflare values of scale-free and multiscale parameters of flaring active regions and the respective peak values of non-flaring regions. Shown are (a) the unsigned magnetic flux $\Phi_{\text {tot }}$, (b) the fractal dimension $D_{0}$, (c) the turbulent power-spectrum index $\alpha$, and (d) the change in the inertial-range scaling exponent $\zeta(3)$.

test gives some distinguishing patterns, the studied parameter provides clues about the expected flare productivity of an active region, without necessarily implying when flares will occur. This claim is found in multiple instances in the literature (e.g., McAteer, Gallagher, and Ireland, 2005 for $D_{0}$; Abramenko, 2005 and Hewett et al., 2008 for $\alpha$; Conlon et al., 2008 for other multifractal parameters).

\subsection{Preflare vs. Peak Non-Flaring Parameter Values}

Figure 5 depicts the preflare values of $\Phi_{\text {tot }}$ (Figure $5 \mathrm{a}$ ), $D_{0}$ (Figure $5 \mathrm{~b}$ ), and $\alpha$ (Figure 5: . It also provides the change between the preflare and the postflare values of $\zeta(3)$ (Figure $5 \mathrm{~d}$ ) for flaring regions, compared with the peak $\zeta(3)$-change for non-flaring ones. Flaring regions are divided into "M-flaring" (regions that have given at least one M-class, but not a X-class, flare; blue squares) and "Xflaring" (regions that have given at least one X-class flare; red squares). Since peaks of the studied parameter timeseries are taken, each active-region timeseries has been inspected separately to remove spurious effects in the parameters' evolution. The most prominent source of these effects is contamination due to flare emission, in the case of large white-light flares, with temporary instrumental or data problems playing a secondary role. In case spurious effects are detected, the affected parameter value is replaced by the value interpolated for a given time.

Inspecting Figure 5, we first notice that only the $\Phi_{\text {tot-values of the flaring }}$ regions (Figure [5]) show some tendency to occupy the upper $\Phi_{\text {tot-range in the }}$ 
plot. The preflare values of the fractal dimension $D_{0}$ (Figure 5 b) appear to be more or less uniformly distributed between $\approx 1.2$ and $\approx 1.8$, with a mean value of $\approx 1.41$ and a standard deviation of $\approx 0.08$. For non-flaring regions, the peak fractal dimension has a mean of $\approx 1.44$, with a standard deviation $\approx 0.14$. Qualitatively, we reproduce the result of McAteer, Gallagher, and Ireland (2005) who found $D_{0} \gtrsim 1.2$ for flaring regions, but we also find that $D_{0} \gtrsim 1.2$ for all active regions in our sample.

A similar behavior is seen when the scaling index $\alpha$ of the turbulent power spectrum is examined (Figure 5r). Indeed, the preflare $\alpha$-values show a mean $\approx 2.4$ and a standard deviation $\approx 0.32$, while the peak $\alpha$-values for the nonflaring regions have a mean $\approx 2.60$ with a standard deviation $\approx 0.42$. No active region shows a $\alpha$-value smaller than the Kolmogorov index of $5 / 3$ and very few regions, among them three M-flaring ones, show $\alpha<2$. We conclude that a strong departure from a Kolmogorov turbulent spectrum is not a characteristic of some (flaring) active regions but one of most active regions. Given the $\alpha$-dependence on spatial resolution, however (Section 3), we cannot be certain about the "true" $\alpha$-value.

For the multifractal inertial-range scaling exponent $\zeta(3)$ we have compared the change in values between the preflare and the postflare phase with the peak change in values for non-flaring active regions (Figure 5d). This was chosen because in Georgoulis (2005) we inferred a significant, permanent decrease in $\zeta(3)$ from preflare to postflare in a single flaring region. Unfortunately, this feature does not survive here, where more comprehensive statistics are involved: for the 111 major flares included in our sample (24 X-class, 87 M-class) the host active regions show a preflare / postflare increase in $\zeta(3)$ in 59 cases, with a decrease between the preflare and postflare $\zeta(3)$-values inferred in the remaining 52 cases. Clearly, the peak $\zeta(3)$ changes do not correspond to flaring active regions with the exception of the two M-flaring regions NOAA AR 9087 and 10596, that show two of the sharpest $\zeta(3)$-decreases in the postflare phase, with amplitudes 0.66 and 0.31 , respectively. If these two regions were studied in isolation and one ignored the $\zeta$-dependence on the spatial resolution, then one might have concluded that large flares indeed relate to sharp decreases in $\zeta(3)$, meaning a decrease of the degree of the photospheric intermittency in the active regions after the flare. Given our spatial-resolution test and the large active-region sample, however, such a conclusion is unjustified.

The apparent failure of the first, stringent test comparing the preflare values of the studied parameters for flaring regions with the respective peak values for non-flaring ones indicates that none of the scale-free and multiscale parameters shows any notable short-term flare prediction capability or, at least, any better predictive capability than the conventional unsigned magnetic flux.

\subsection{Peak Flaring vs. Peak Non-Flaring Parameter Values}

We now perform the second test by comparing the peak values of the studied parameters for both flaring and for non-flaring regions. The results of this test are shown in Figure 6 .

Figure 6a depicts the results for the unsigned magnetic flux $\Phi_{\text {tot }}$. It shows more clearly than Figure 5 a that the peak $\Phi_{\text {tot-values tend to occupy the upper }}$ 

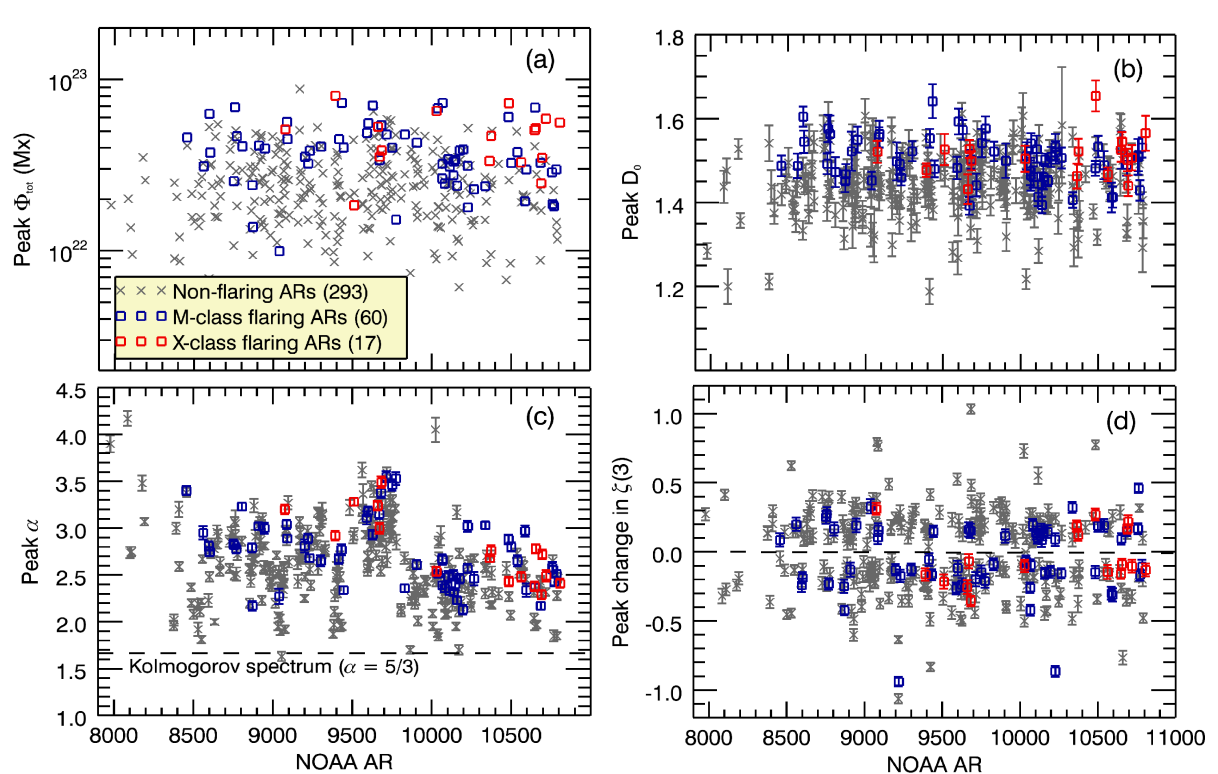

Figure 6. Same as Figure [5 but showing the peak, rather than the preflare, values of the examined parameters for the flaring active regions.

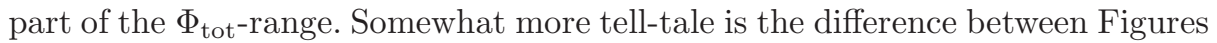
$5 \mathrm{~b}$ and $6 \mathrm{~b}$ in terms of the preflare and the peak fractal dimension, respectively: the peak $D_{0}$-values for flaring regions in Figure $6 \mathrm{~b}$ also tend to occupy the upper part of the $D_{0}$-range, meaning that flaring regions statistically tend to have higher fractal dimension, as McAteer, Gallagher, and Ireland (2005) previously reported.

Regarding the indices $\alpha$ of the turbulent power spectrum (Figure 6r), there is no distinct difference with Figure 5 . The peak $\alpha$-values for flaring regions also tend to occupy the higher $\alpha$-range but with larger dispersion than the fractal dimension $D_{0}$. This being said, the active regions with the highest peak $\alpha$-values in our sample happen to be non-flaring ones.

When the peak $\zeta(3)$ change for both flaring and non-flaring regions is considered (Figure 6 $6 \mathrm{~d}$ ) we find that flaring regions show a similar pattern with non-flaring ones. Only for the X-flaring regions there seems to be a weak statistical preference for stronger $\zeta(3)$-decreases as compared to increases, with a ratio 11:6. The probability that this preference is by chance, however, is rather high, of the order 0.09 . This probability was calculated by means of a binomial probability function, assigning a 0.5 probability that the peak $\zeta(3)$-change will be positive and assuming 11 positive chance "hits" out of 17 independent trials (since the $\zeta(3)>0$ probability is 0.5 the binomial distribution becomes symmetric with respect to chance hits, so identical results would be reached in case we assumed 6 negative chance "hits" out of 17 trials). For M-flaring regions, stronger $\zeta(3)$-decreases barely dominate, with a ratio $32: 28$. This time, however, the binomial probability that this result is random is $\approx 3 \times 10^{-14}$, so we can safely conclude that there is no clear preference of $\zeta(3)$-decreases over increases 
Table 2. Summary of means and standard deviations (in parentheses) for the preflare and peak values of $\Phi_{\text {tot }}$, the scale-free $D_{0}$, and multiscale parameters $\alpha$ and $\zeta(3)$, calculated in our sampl of $17 \mathrm{X}$-flaring, $60 \mathrm{M}$-flaring, and 293 non-flaring active regions. The preflare active-region value correspond to $24 \mathrm{X}$-class and $87 \mathrm{M}$-class flares. The discrepancy between numbers of flaring region and flares is because some flaring regions flare repeatedly over the observing interval.

\begin{tabular}{lccccc}
\hline & \multicolumn{2}{c}{ Preflare values } & \multicolumn{3}{c}{ Peak values } \\
Active Regions & X-flaring & M-flaring & X-flaring & M-flaring & Non-flaring \\
\hline Data points & 24 & 87 & 17 & 60 & 293 \\
$\Phi_{\text {tot }}\left(\times 10^{22} M x\right)$ & $4.57(1.63)$ & $3.55(1.72)$ & $4.74(1.68)$ & $3.84(1.56)$ & $2.58(1.21)$ \\
$D_{0}$ & $1.44(0.07)$ & $1.40(0.08)$ & $1.51(0.05)$ & $1.50(0.05)$ & $1.44(0.13)$ \\
$\alpha$ & $2.38(0.29)$ & $2.40(0.33)$ & $2.77(0.36)$ & $2.73(0.36)$ & $2.60(0.42)$ \\
$\zeta(3)$-change & $0.012(0.06)$ & $-0.001(0.11)$ & $-0.03(0.20)$ & $-0.04(0.26)$ & $-0.004(0.29)$ \\
\hline
\end{tabular}

in case of M-flaring regions. For non-flaring regions, the peak $\zeta(3)$-changes are almost evenly divided between decreases and increases, with a ratio 146:147, that is again not random (the binomial probability that this result is random, given the large sample size, is practically zero). Overall, it becomes clear that one cannot use the preflare (Figure 5b) or the peak (Figure 6b) change in $\zeta(3)$ to assess the flaring productivity of an active region, meaning that flaring regions do not show distinguishable, sharp changes in their degree of intermittency.

Table 2 quantifies Figures 5 and 6, providing means and standard deviations for each depicted distribution. It shows that only the peak values of the unsigned flux $\Phi_{t o t}$ and the fractal dimension $D_{0}$ are somewhat different between flaring and non-flaring regions but the respective dispersions are such that there is considerable mixing of values between the three active-region populations. For the preflare values of $\Phi_{t o t}$ and $D_{0}$ there is even more mixing and, in terms of $D_{0}, \mathrm{X}$ - and M-flaring regions are practically indistinguishable. When it comes to $\alpha$-values and changes in $\zeta(3)$, Table 2 - along with Figures 5 and 6 - shows that these multiscale parameters simply cannot be used to distinguish flaring from non-flaring regions, let alone predict large flares within a given time span.

\subsection{Flare Forecasting Probabilities}

In Sections 4.1, 4.2 we found that neither the preflare nor the peak values of our scale-free and multiscale parameters seem capable of distinguishing flaring from non-flaring regions. The unsigned flux $\Phi_{\text {tot }}$ tends to score better than the scaleinvariant $D_{0}$, the multiscale $\alpha$, and the change in $\zeta(3)$. To further quantify these results we calculate here the conditional probability of an active region being a flaring one if a given parameter inferred from any one of its magnetograms exceeds a preset threshold. A way to do this is by using Laplace's rule of succession and proceeding to a Bayesian inference of the predictive probability as follows: assume that $F$ magnetograms of flaring regions and $N$ magnetograms of non-flaring ones exhibit a value $R$ of a parameter that exceeds a threshold $R_{\text {thres }}$. Then, the conditional probability $p$ that an active-region with $R>R_{\text {thres }}$ 


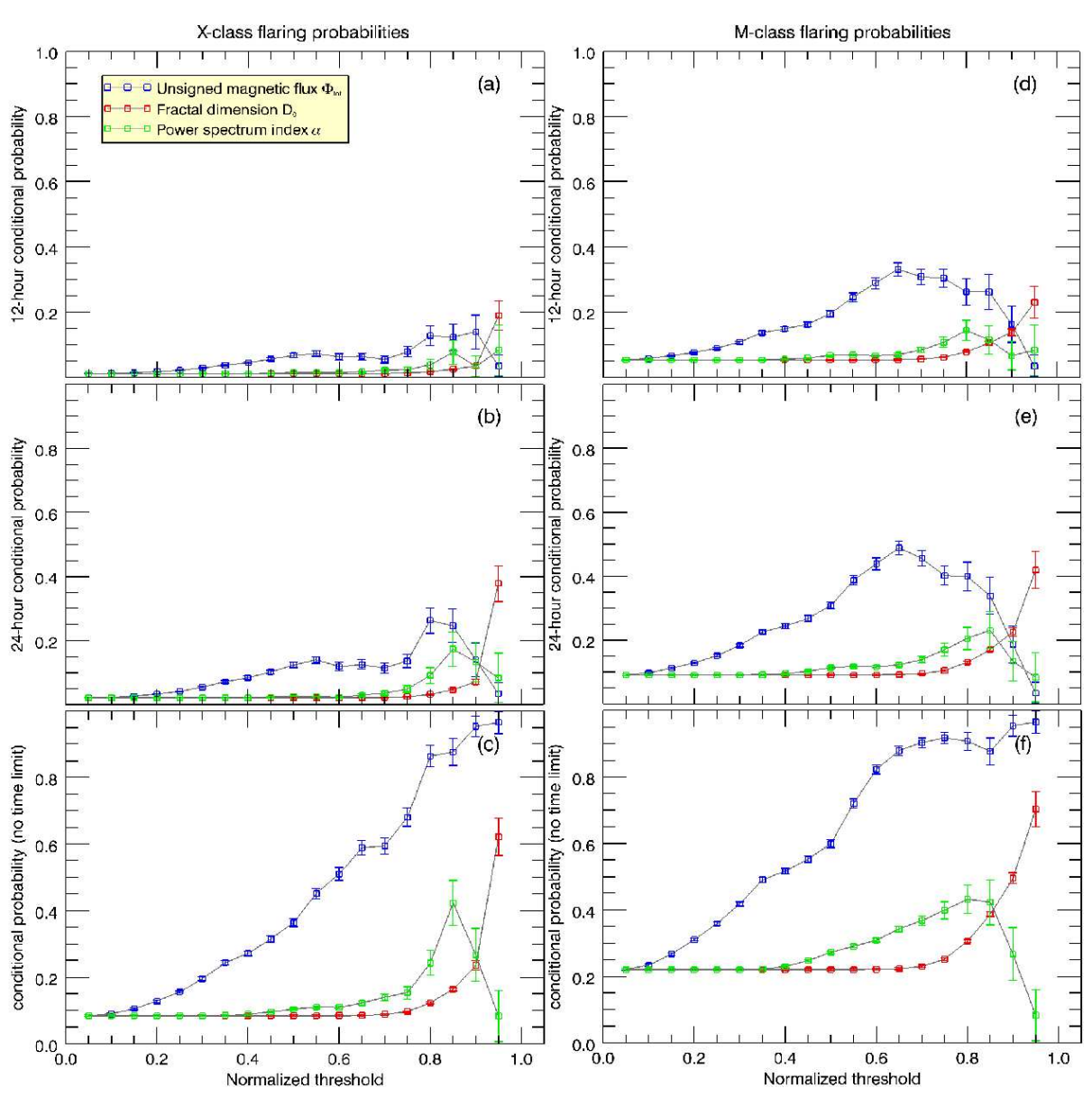

Figure 7. Conditional probabilities of active regions to host a major flare, either X-class (left column) or M-class (right column), with respect to the normalized (against the maximum) threshold of a given parameter, with $\Phi_{\text {tot }}$ (blue squares), $D_{0}$ (red squares), and $\alpha$ (green squares) examined. The upper (a, d) and middle (b, e) rows provide the 12- and 24-hour conditional probabilities, respectively. The lower row (c, f) provides the probability without a time limit, that is, the probability of major flaring at a future time when the active region is still visible in the disk. The maximum values against which the thresholds were normalized are $8.8 \times 10^{22} \mathrm{Mx}, 1.65$, and 4.17 for $\Phi_{\text {tot }}, D_{0}$, and $\alpha$, respectively.

will be a flaring one is given by (Jaynes, 2003, pp. 155-156)

$$
p=\frac{F+1}{N+2} \quad \text { with } \quad \text { uncertainty } \quad \delta p=\sqrt{\frac{p(1-p)}{N+3}} .
$$

This probability rule was also used by Wheatland (2005) to test another solarflare prediction method. To compare directly between the conditional probabilities of the various parameters, we normalize the thresholds with respect to the maximum value of each parameter appearing in Figures 5 and 6. We examine only three of the four parameters - $\Phi_{\text {tot }}, D_{0}$, and $\alpha$ - because the changes of $\zeta(3)$ obviously show very similar patterns for flaring and non-flaring regions. Using 
the NOAA/GOES and Yohkoh/HXT flare catalogs (Section 2.1), we consider a given active-region magnetogram as a preflare one if a major flare happened in the region within a given, preset timeframe from the time that the magnetogram was recorded. Figure 7 shows conditional probabilities for two timeframes: 12 hours (Figure $7 \mathrm{~b}, \mathrm{~d}$ ) and 24 hours (Figure $7 \mathrm{~b}, \mathrm{e}$ ), while Figures $7 \mathrm{f}$ and f show conditional probabilities without any timeframe assigned: if an active region flared at any time after the magnetogram was taken, then this magnetogram is considered a preflare one. Notice that the flares under examination may have occurred when a given region has moved beyond the $30^{\circ} \mathrm{E}-\mathrm{W}$ meridional zone of analysis. This does not affect the analysis, however, as the preflare magnetograms were acquired when the region was still within the analysis zone.

All plots of Figure 7 illustrate that the unsigned flux $\Phi_{t o t}$, a conventional activity predictor, is generally more effective in predicting major flares than both the scale-free fractal dimension $D_{0}$ and the multiscale turbulent power-spectrum index $\alpha$. Differences between $\Phi_{\text {tot }}$ and $\left(D_{0}, \alpha\right)$ are smaller (but also reflect small flare probabilities, of limited practical use) in case of the most demanding prediction, the one with a 12-hour timeframe for flares $>$ X1.0 (Figure $7 \mathrm{a}$ ). In all other cases $\Phi_{\text {tot }}$ gives much higher (well beyond error bars) probabilities than $D_{0}$ and $\alpha$. The predictive ability of $D_{0}$ appears comparable with, or slightly higher than, that of $\Phi_{\text {tot }}$ only for normalized thresholds $R_{\text {thres }} \geq 0.9$ for the 12 - and 24-hour prediction timeframes. For the same timeframes, the predictive ability of $\Phi_{\text {tot }}$ drops for $R_{\text {thres }} \gtrsim 0.8$ (Figures $7 \mathrm{a}, \mathrm{b}$ ) and $R_{\text {thres }} \gtrsim 0.6$ (Figures $7 \mathrm{~d}, \mathrm{e}$ ). This is because the upper $\Phi_{t o t}$-ranges in these cases are occupied by non-flaring (within the preset timeframes) active regions - this is one of the limitations for using the unsigned flux as a flare predictor. The power-spectrum index $\alpha$ exhibits similar behavior, but for higher $R_{\text {thres }} \gtrsim 0.8$, in all plots of Figure 7. Comparing the multiscale $\alpha$ with the scale-free $D_{0}$, we note that $\alpha$ works somewhat better, especially for larger timeframes. This is in line with Abramenko s (2005) suggestion that $\alpha$ better reflects future flare productivity. However, recall that $\alpha$ depends sensitively on the spatial resolution of the observing instrument, contrary to $D_{0}$ (Table 1 ). Hence the results of Figure 7 concerning $\alpha$ should be viewed as holding exclusively for MDI low-resolution magnetograms.

\section{Summary and Conclusion}

This study investigates previous claims on the efficiency of fractal and multifractal techniques as reliable predictors of major solar flares and/or parameters reflecting the overall flare productivity of solar active regions before they actually flare. From the array of parameters implemented in the literature, we select three of the reported most promising ones: the fractal dimension, the multifractal intermittency index, and the scaling index of the turbulent power spectrum. Our objective is not to judge the methods per se but, rather, to test the notion of utilizing fractality and multifractality to gain predictive insight into major solar flares.

Statistical analyses such as this one must guarantee that the assembled activeregion sample is representative: the sample must contain numerous flaring and 
non-flaring regions. Comprehensive statistics often help avoid the interpretation of incidental signals as statistically significant behavior. Section 4.1 (Figure 5 $\mathrm{d}$ ) includes examples of results that might have been interpreted in a misleading way had the statistics of our active-region sample been insufficient.

We study $370 \mathrm{SOHO} / \mathrm{MDI}$ low-resolution (1.98" per pixel) timeseries of active-region magnetograms, 293 of which correspond to active regions without major flares and 77 correspond to M- and X-class flaring regions. MDI line-of-sight fields are used for regions within $30^{\circ}$ of the central meridian in order to approximate the longitudinal-field component with the normal-field component and avoid any corrections or otherwise modifications of the original MDI data. We find that neither scale-free (fractal) nor multiscale (multifractal) techniques can be used to predict major flares, or for the a priori assessment of the flaring productivity of active regions. In particular, we find that their diagnostic capability is not better than that of the unsigned magnetic flux of active regions, a traditional, but unreliable, activity predictor. Since the fractal and multifractal measures tested here are less effective than the unsigned flux (Figure 7), they should not be used for flare prediction or for flaring productivity assessment.

On the fundamental question of whether flaring active regions are more fractal, multifractal, or turbulent than other, non-flaring ones, the answer per our results has to be negative: flaring regions tend to exhibit relatively large peak values of scale-free and multiscale parameters but these values, or even higher ones sometimes, are also exhibited by non-flaring regions. For all statistical distributions, the means and standard deviations are such that the different populations of flaring and non-flaring regions overlap considerably (Table 2).

At this point we emphasize our willingness to follow the guidelines of multiple previous studies in the inference of the above fractal and multifractal parameters. In particular, we followed McAteer, Gallagher, and Ireland (2005) when inferring the fractal dimension $D_{0}$, Abramenko (2005) when inferring the turbulent scaling index $\alpha$ (despite the fact that Abramenko worked exclusively on high-resolution MDI magnetograms), and a previous work of this author (Georgoulis, 2005), together with Abramenko et al. (2003), when inferring the intermittency index $\zeta(q)$. As a result, the findings of both McAteer, Gallagher, and Ireland (2005) and Abramenko (2005) were qualitatively reproduced in this analysis, while we showed that the distinct $\zeta(3)$-behavior reported by Georgoulis (2005) was just one incidental case and not part of a systematic trend.

In addition, this work (Section 3) exposes a dependence of multiscale parameters $\zeta(q)$ and $\alpha$ on the spatial resolution of the studied magnetograms. In contrast, the scale-free $D_{0}$ appears fairly insensitive to varying spatial resolution. Therefore, results and comparisons for $\zeta(3)$ and $\alpha$ in Section 4 are valid only for MDI low-resolution data and should not be generalized to data sets of other instruments. Possible susceptibility of the $D_{0}$-value should also be studied with respect to the threshold it requires, unlike $\zeta(3)$ and $\alpha$. This investigation has not been carried out here. In previous works, however, Meunier (1999) reported a decreasing trend of $D_{0}$ with increasing threshold, while Janßen, Vögler, and Kneer (2003) reported a slighter decrease, or a near insensitivity, of $D_{0}$ for increasing thresholds, in case these thresholds are sufficiently above noise levels or the magnetic field data have been treated for noise, respectively. 
It is useful to mention here a very recent result by Abramenko and Yurchyshyn (2010) that the turbulent power-spectrum index $\alpha$, either alone or coupled with the integral of the power-spectrum for all wavenumbers, correlates better than $\Phi_{\text {tot }}$ with the flaring index in a large sample of 217 active regions recorded in high-resolution MDI magnetograms. While correlating some parameter with the flaring index is not identical to inferring the predictive capability of this parameter, these results appear in likely contrast with the results presented here. Further investigation is clearly needed, therefore. Nonetheless, some convergence of views appears in that multiscale parameters may not be ideal tools for solar flare prediction (Abramenko, 2010, private communication).

Perhaps more instructive than pointing out the inability of scale-free and multiscale techniques to assess a priori the flaring record of active regions is to explain why this is the case. In this author's view, there are at least two distinct reasons that justify our findings:

First, fractality and multifractality are extremely widespread in the solar atmosphere, eruptive and quiescent alike. This may well be due to the turbulence dominating the magnetic-flux generation and emergence process (see Introduction). For example, recall the fractality of white-light granules (Roudier and Muller, 1987: Hirzberger et al., 1997), the fractality and multifractality of active regions and the quiet-Sun magnetic field (Schrijver et al., 1992; Cadavid et al., 1994: Meunier, 1999 Janßen, Vögler, and Kneer, 2003), the fractality of flares and sub-flares in the EUV (Aschwanden and Parnell, 2002; Aschwanden and Aschwanden, 2008a; 2008b), the fractality of the quiet network in the EUV (Gallagher et al., 1998), that of Ellerman bombs in off-band $\mathrm{H} \alpha$ (Georgoulis et al., 2002), and others. The fractal dimension in most, if not all, of these works varies between 1.4 and 1.8 , practically indistinguishable from the fractal dimension of active regions found here. As a result, it appears unlikely that these same methods may reflect particular characteristics of active regions, let alone flare productivity.

Second, there is a lack of correlations between the fractal dimension in the photosphere and that of the overlying chromosphere and corona, where major flares occur. Dimitropoulou et al. (2009) assumed nonlinear force-free magnetic fields extending above the photosphere and calculated volumes of enhanced electric currents and steep magnetic gradients from these extrapolated fields. They found no correlation between the three-dimensional fractal dimension of these volumes and that of the two-dimensional photospheric boundary. In other words, all photospheric "memory", in terms of fractality and multifractality, is erased above the photosphere due to the fact that these unstable volumes become nearly space-filling slightly above this boundary. Attempting to assess the fractality of layers higher than the photosphere - where flares occur - by using the photospheric fractality as a proxy will not yield meaningful results, similarly to the lack of correlation between photospheric electric currents and the coronal X-ray brightness (Metcalf et al., 1994).

In addition, it is possible that both flaring and non-flaring regions share a similar degree of self-organization in the distribution of their magnetic free energy, as reported by Vlahos and Georgoulis (2004). Flaring regions have an "opportunity" to show their self-organization via flaring, with flares inheriting the statistics of their host active regions, while non-flaring regions retain this 
property without demonstrating it. In this sense i) fractality alone cannot be responsible for flaring, and ii) fractality, as a global characteristic of the activeregion atmosphere, cannot be used to determine a priori which active regions will flare.

There are, of course, sophisticated multiscale techniques not treated in this work, such as wavelet methods used to extract the magnetic-energy spectrum in active regions (Hewett et al., 2008) or to distinguish active regions from quiet Sun for further treatment (Conlon et al., 2010), or the flatness function and its intermittency index (Abramenko, Yurchyshyn, and Wang, 2008). While we cannot comment on methods that we have not tested, per our conclusions it would seem rather surprising if a scale-free or multiscale technique delivered a notable improvement in our forecasting ability, as this would apparently contradict what scale-free and multiscale behavior caused by self-organization is meant to imply: spontaneity in the system's dynamical response to external forcing, both in timing and in amplitude, and hence a lack of certainty in predicting this response.

Let us finally mention that alternative flare prediction approaches have been developed in recent years. Rather than fractality, multifractality, or intermittency and turbulence, these methods rely on parameters stemming from morphological and topological characteristics of active regions, such as those of the photospheric magnetic-polarity inversion lines or photospheric properties in general (Falconer, Moore, and Gary, 2006, Schrijver, 2007; Georgoulis and Rust, 2007, Leka and Barnes, 2007; Mason and Hoeksema, 2010), or those of the subsurface kinetic helicity prior to active-region emergence (Reinard et al., 2010), among others. It remains to be seen whether these parameters can lead to advances in the forecasting of major solar eruptions or whether forecasting will remain inherently probabilistic which, per our results, seems entirely possible. In any case, fractal and multifractal methods - perhaps not extremely useful as eruption predictors - will always be excellent tools for a fundamental understanding of the origins and nature of solar magnetism.

Acknowledgements This work is based on a talk given by the author during the Fourth Solar Image Processing (SIP) Workshop in Baltimore, MD, USA, 26-30 October 2008. Thanks are due to the organizers for an interesting and productive meeting. During the author's tenure at the Johns Hopkins University Applied Physics Laboratory (JHU/APL) in Laurel, MD, USA, this work received partial support from NASA's LWS TR\&T Grant NNG05GM47G and Guest Investigator Grant NNX08AJ10G. The author gratefully acknowledges the Institute of Space Applications and Remote Sensing (ISARS) of the National Observatory of Athens for the availability of their computing cluster facility for massive runs related to this work. SOHO is a project of international cooperation between ESA and NASA. Hinode is a Japanese mission developed and launched by ISAS/JAXA, with NAOJ as domestic partner and NASA and STFC (UK) as international partners. It is operated by these agencies in co-operation with ESA and NSC (Norway). Finally, the author thanks the two anonymous referees for contributing to the clarity, accuracy, and focus of this work.

\section{References}

Abramenko, V., Yurchyshyn, V.: 2010, Intermittency and Multifractality Spectra of the Magnetic Field in Solar Active Regions. Astrophys. J. 722, $122-130$. doi $10.1088 / 0004-637 X / 722 / 1 / 122$ 
Abramenko, V., Yurchyshyn, V., Wang, H.: 2008, Intermittency in the Photosphere and Corona above an Active Region. Astrophys. J. 681, 1669-1676. doi 10.1086/588426

Abramenko, V.I.: 2005, Relationship between Magnetic Power Spectrum and Flare Productivity in Solar Active Regions. Astrophys. J. 629, 1141-1149. doi $10.1086 / 431732$

Abramenko, V.I., Yurchyshyn, V.B., Wang, H., Spirock, T.J., Goode, P.R.: 2002, Scaling Behavior of Structure Functions of the Longitudinal Magnetic Field in Active Regions on the Sun. Astrophys. J. 577, 487-495. doi 10.1086/342169

Abramenko, V.I., Yurchyshyn, V.B., Wang, H., Spirock, T.J., Goode, P.R.: 2003, Signature of an Avalanche in Solar Flares as Measured by Photospheric Magnetic Fields. Astrophys. J. 597, 1135-1144. doi 10.1086/378492

Aschwanden, M.J., Aschwanden, P.D.: 2008a, Solar Flare Geometries. I. The Area Fractal Dimension. Astrophys. J. 674, 530-543. doi $10.1086 / 524371$

Aschwanden, M.J., Aschwanden, P.D.: 2008b, Solar Flare Geometries. II. The Volume Fractal Dimension. Astrophys. J. 674, 544-553. doi $10.1086 / 524370$

Aschwanden, M.J., Parnell, C.E.: 2002, Nanoflare Statistics from First Principles: Fractal Geometry and Temperature Synthesis. Astrophys. J. 572, $1048-1071$. doi $10.1086 / 340385$.

Bak, P., Tang, C., Wiesenfeld, K.: 1987, Self-organized criticality - An explanation of 1/f noise. Physical Review Letters 59, 381 - 384. doi 10.1103/PhysRevLett.59.381

Bak, P.: 1996, How nature works : The science of self-organized criticality, Copernicus Press, New York, NY, USA.

Berger, T.E., Lites, B.W.: 2003, Weak-Field Magnetogram Calibration using Advanced Stokes Polarimeter Flux Density Maps - II. SOHO/MDI Full-Disk Mode Calibration. Solar Phys. 213, 213-229.

Biskamp, D., Welter, H.: 1989, Dynamics of decaying two-dimensional magnetohydrodynamic turbulence. Physics of Fluids B 1, 1964-1979. doi:10.1063/1.859060

Brandenburg, A., Tuominen, I., Nordlund, A., Pulkkinen, P., Stein, R.F.: 1990, 3-D simulation of turbulent cyclonic magneto-convection. Astron. Astrophys. 232, $277-291$.

Cadavid, A.C., Lawrence, J.K., Ruzmaikin, A.A., Kayleng-Knight, A.: 1994, Multifractal models of small-scale solar magnetic fields. Astrophys. J. 429, 391-399. doi $10.1086 / 174329$.

Cattaneo, F., Emonet, T., Weiss, N.: 2003, On the Interaction between Convection and Magnetic Fields. Astrophys. J. 588, 1183-1198. doi 10.1086/374313

Conlon, P.A., Gallagher, P.T., McAteer, R.T.J., Ireland, J., Young, C.A., Kestener, P., Hewett, R.J., Maguire, K.: 2008, Multifractal Properties of Evolving Active Regions. Solar Phys. 248, 297 - 309. doi:10.1007/s11207-007-9074-7

Conlon, P.A., McAteer, R.T.J., Gallagher, P.T., Fennell, L.: 2010, Quantifying the Evolving Magnetic Structure of Active Regions. Astrophys. J. 722, 577-585. doi $10.1088 / 0004-637 \mathrm{X} / 722 / 1 / 577$

Dimitropoulou, M., Georgoulis, M., Isliker, H., Vlahos, L., Anastasiadis, A., Strintzi, D., Moussas, X.: 2009, The correlation of fractal structures in the photospheric and the coronal magnetic field. Astron. Astrophys. 505, 1245-1253. doi $10.1051 / 0004-6361 / 200911852$

Evertsz, C.J.G., Mandelbrot, B.B.: 1992, Self-similarity of harmonic measure on DLA. Physica A Statistical Mechanics and its Applications 185, $77-86$. doi $10.1016 / 0378-4371(92) 90440-2$

Falconer, D.A., Moore, R.L., Gary, G.A.: 2006, Magnetic Causes of Solar Coronal Mass Ejections: Dominance of the Free Magnetic Energy over the Magnetic Twist Alone. Astrophys. J. 644, $1258-1272$. doi $10.1086 / 503699$

Fragos, T., Rantsiou, E., Vlahos, L.: 2004, On the distribution of magnetic energy storage in solar active regions. Astron. Astrophys. 420, 719-728. doi 10.1051/0004-6361:20034570.

Frisch, U.: 1995, Turbulence. The legacy of A.N. Kolmogorov, Cambridge University Press, Cambridge, UK.

Gallagher, P.T., Phillips, K.J.H., Harra-Murnion, L.K., Keenan, F.P.: 1998, Properties of the quiet Sun EUV network. Astron. Astrophys. 335, 733-745.

Georgoulis, M., Kluiving, R., Vlahos, L.: 1995, Extended instability criteria in isotropic and anisotropic energy avalanches. Physica A Statistical Mechanics and its Applications 218, $191-213$

Georgoulis, M.K.: 2005, Turbulence In The Solar Atmosphere: Manifestations And Diagnostics Via Solar Image Processing. Solar Phys. 228, 5-27. doi:10.1007/s11207-005-2513-4

Georgoulis, M.K., Rust, D.M.: 2007, Quantitative Forecasting of Major Solar Flares. Astrophys. J. Lett. 661, L109-L112. doi 10.1086/518718.

Georgoulis, M.K., Raouafi, N.E., Henney, C.J.: 2008, Automatic Active-Region Identification and Azimuth Disambiguation of the SOLIS/VSM Full-Disk Vector Magnetograms. In: 
Howe, R., Komm, R.W., Balasubramaniam, K.S., Petrie, G.J.D. (eds.) Subsurface and Atmospheric Influences on Solar Activity, Astronomical Society of the Pacific Conference Series 383, 107-114.

Georgoulis, M.K., Rust, D.M., Bernasconi, P.N., Schmieder, B.: 2002, Statistics, Morphology, and Energetics of Ellerman Bombs. Astrophys. J. 575, 506-528. doi 10.1086/341195

Hewett, R.J., Gallagher, P.T., McAteer, R.T.J., Young, C.A., Ireland, J., Conlon, P.A., Maguire, K.: 2008, Multiscale Analysis of Active Region Evolution. Solar Phys. 248, 311 - 322. doi $10.1007 /$ s11207-007-9028-0

Hirzberger, J., Vazquez, M., Bonet, J.A., Hanslmeier, A., Sobotka, M.: 1997, Time Series of Solar Granulation Images. I. Differences between Small and Large Granules in Quiet Regions. Astrophys. J. 480, 406. doi 10.1086/303951

Hurlburt, N.E., Brummel, N.H., Toomre, J.: 1995, Local-Area Simulations of Rotating Compressible Convection and Associated Mean Flows. In: Hoeksema, J.T., Domingo, V., Fleck, B., Battrick, B. (eds.) Helioseismology, ESA Special Publication 376, 245-248.

Janßen, K., Vögler, A., Kneer, F.: 2003, On the fractal dimension of small-scale magnetic structures in the Sun. Astron. Astrophys. 409, 1127-1134. doi 10.1051/0004-6361:20031168.

Jaynes, E.T.: 2003, Probability Theory: The Logic of Science, Cambridge University Press, Cambridge, UK.

Kluiving, R., Pasmanter, R.A.: 1996, Stochastic selfsimilar branching and turbulence. Physica A Statistical Mechanics and its Applications 228, 273-294.

Kolmogorov, A.: 1941, The Local Structure of Turbulence in Incompressible Viscous Fluid for Very Large Reynolds' Numbers. Akademiia Nauk SSSR Doklady 30, 301-305.

Kraichnan, R.H.: 1965, Inertial-Range Spectrum of Hydromagnetic Turbulence. Physics of Fluids 8, $1385-1387$. doi 10.1063/1.1761412

LaBonte, B.J., Georgoulis, M.K., Rust, D.M.: 2007, Survey of Magnetic Helicity Injection in Regions Producing X-Class Flares. Astrophys. J. 671, 955-963. doi $10.1086 / 522682$

Lawrence, J.K., Ruzmaikin, A.A., Cadavid, A.C.: 1993, Multifractal Measure of the Solar Magnetic Field. Astrophys. J. 417, 805. doi 10.1086/173360

Leka, K.D., Barnes, G.: 2003, Photospheric Magnetic Field Properties of Flaring versus Flare-quiet Active Regions. II. Discriminant Analysis. Astrophys. J. 595, 1296-1306. doi $10.1086 / 377512$

Leka, K.D., Barnes, G.: 2007, Photospheric Magnetic Field Properties of Flaring versus Flarequiet Active Regions. IV. A Statistically Significant Sample. Astrophys. J. 656, 11731186. doi $10.1086 / 510282$

Lites, B.W., Elmore, D.F., Streander, K.V.: 2001, The Solar-B Spectro-Polarimeter. In: Sigwarth, M. (ed.) Advanced Solar Polarimetry - Theory, Observation, and Instrumentation, Astronomical Society of the Pacific Conference Series 236, 33-40.

Longcope, D.W., Fisher, G.H., Pevtsov, A.A.: 1998, Flux-Tube Twist Resulting from Helical Turbulence: The Sigma-Effect. Astrophys. J. 507, 417-432. doi $10.1086 / 306312$

Mandelbrot, B.B.: 1983, The fractal geometry of nature/Revised and enlarged edition/, W. H. Freeman and Co., New York, NY, USA.

Mason, J.P., Hoeksema, J.T.: 2010, Testing Automated Solar Flare Forecasting with 13 Years of Michelson Doppler Imager Magnetograms. Astrophys. J. 723, 634-640. doi $10.1088 / 0004-637 X / 723 / 1 / 634$

McAteer, R.T.J., Gallagher, P.T., Conlon, P.A.: 2010, Turbulence, complexity, and solar flares. Adv. Space Res. 45, 1067 -1074. doi 10.1016/j.asr.2009.08.026.

McAteer, R.T.J., Gallagher, P.T., Ireland, J.: 2005, Statistics of Active Region Complexity: A Large-Scale Fractal Dimension Survey. Astrophys. J. 631, 628-635. doi 10.1086/432412.

Metcalf, T.R., Canfield, R.C., Hudson, H.S., Mickey, D.L., Wulser, J.P., Martens, P.C.H., Tsuneta, S.: 1994, Electric currents and coronal heating in NOAA active region 6952. Astrophys. J. 428, $860-866$. doi $10.1086 / 174295$

Meunier, N.: 1999, Fractal Analysis of Michelson Doppler Imager Magnetograms: A Contribution to the Study of the Formation of Solar Active Regions. Astrophys. J. 515, 801-811. doi $10.1086 / 307050$

Nicolis, G., Prigogine, I.: 1989, Exploring complexity. An introduction, W. H. Freeman, New York, NY, USA.

Reinard, A.A., Henthorn, J., Komm, R., Hill, F.: 2010, Evidence That Temporal Changes in Solar Subsurface Helicity Precede Active Region Flaring. Astrophys. J. Lett. 710, L121 - L125. doi 10.1088/2041-8205/710/2/L121

Roudier, T., Muller, R.: 1987, Structure of the solar granulation. Solar Phys. 107, 11-26. 
Scherrer, P.H., Bogart, R.S., Bush, R.I., Hoeksema, J.T., Kosovichev, A.G., Schou, J., Rosenberg, W., Springer, L., Tarbell, T.D., Title, A., Wolfson, C.J., Zayer, I., MDI Engineering Team: 1995, The Solar Oscillations Investigation - Michelson Doppler Imager. Solar Phys. 162, $129-188$. doi:10.1007/BF00733429

Schrijver, C.J.: 2007, A Characteristic Magnetic Field Pattern Associated with All Major Solar Flares and Its Use in Flare Forecasting. Astrophys. J. Lett. 655, L117-L120. doi $10.1086 / 511857$

Schrijver, C.J., Zwaan, C., Balke, A.C., Tarbell, T.D., Lawrence, J.K.: 1992, Patterns in the photospheric magnetic field and percolation theory. Astron. Astrophys. 253, L1-L4.

Schroeder, M.: 1991, Fractals, chaos, power laws. minutes from an infinte paradise, Freeman, New York, NY, USA.

Seiden, P.E., Wentzel, D.G.: 1996, Solar Active Regions as a Percolation Phenomenon. II. Astrophys. J. 460, 522. doi 10.1086/176989

Vlahos, L., Georgoulis, M.K.: 2004, On the Self-Similarity of Unstable Magnetic Discontinuities in Solar Active Regions. Astrophys. J. Lett. 603, L61-L64. doi 10.1086/383032

Vlahos, L., Fragos, T., Isliker, H., Georgoulis, M.: 2002, Statistical Properties of the Energy Release in Emerging and Evolving Active Regions. Astrophys. J. Lett. 575, L87-L90. doi $10.1086 / 342826$

Wentzel, D.G., Seiden, P.E.: 1992, Solar active regions as a percolation phenomenon. Astrophys. J. 390, 280-289. doi $10.1086 / 171278$

Wheatland, M.S.: 2005, Initial Test of a Bayesian Approach to Solar Flare Prediction. Publications of the Astronomical Society of Australia 22, 153-156. doi 10.1071/AS04062.

Zhou, G., Wang, J., Wang, Y., Zhang, Y.: 2007, Quasi-Simultaneous Flux Emergence in the Events of October November 2003. Solar Phys. 244, 13-24. doi $10.1007 /$ s11207-007-9032-4. 
SOLA_ms.tex; 23 May 2022; $22: 41 ;$ p. 26 